\title{
Surfactant-Influenced Gas-Liquid Interfaces: Nonlinear Equation of State and Finite Surface Viscosities
}

\author{
Juan M. Lopez ${ }^{*}, 1$ and Amir H. Hirsa $†$ \\ * Department of Mathematics, Arizona State University, Tempe, Arizona 85287; and $\dagger$ Department of Mechanical Engineering, Aeronautical Engineering, \\ and Mechanics, Rensselaer Polytechnic Institute, Troy, New York 12180 \\ Received February 24, 2000; accepted June 5, 2000
}

\begin{abstract}
A canonical flow geometry was utilized for a fundamental study of the coupling between bulk flow and a Newtonian gas-liquid interface in the presence of an insoluble surfactant. We develop a $\mathrm{N}$ avier-Stokes numerical model of the flow in the deep-channel surface viscometer geometry, which consists of stationary inner and outer cylinders, a floor rotating at a constant angular velocity, and an interface covered initially by a uniformly distributed surfactant. $H$ ere, the floor of the annular channel is rotated fast enough so the flow is nonlinear and drives the film toward the inner cylinder. The boundary conditions at the interface are functions of the surface tension, surface shear viscosity, and surface dilatational viscosity, as described by the Boussinesq-Scriven surface model. A physical surfactant system, namely hemicyanine, an insoluble monolayer on an air-water interface, with measured values of surface tension and surface shear viscosity versus concentration, was used in this study. We find that a surfactant front can form, depending on the $R$ eynolds number and the initial surfactant concentration. The stress balance in the radial direction was found to be dominated by the Marangoni stress, but the azimuthal stress was only due to the surface shear viscosity. Numerical studies are presented comparing results of surfactant-influenced interface cases implementing the derived viscoelastic interfacial stress balance with those using a number of idealized stress balances, as well as a rigid no-slip surface, providing added insight into the altered dynamics that result from the presence of a surfactant monolayer. $\odot 2000$ Academic Press
\end{abstract}

Key Words: insoluble surfactants; nonlinear equation of state; surface shear viscosity; contamination front; deep-channel surface viscometer.

\section{INTRODUCTION}

Flow of liquid in systems with a gas-liquid interface can be significantly influenced by the presence of surfactant monolayers. This is a result of the liquid's viscosity, which ensures that the surfactant film at the interface has the same velocity as the fluid at that interface. This hydrodynamic coupling between the fluid and a Newtonian interface (1-3) is a result of the shear stress in the liquid evaluated at the interface, being balanced by (i) Marangoni interfacial stress, due to gradients in surface

\footnotetext{
${ }^{1}$ To whom correspondence should be addressed. E-mail: lopez@math.la.asu. edu, hirsaa@ rpi.edu.
}

tension, (ii) interfacial stress due to surface shear viscosity, and (iii) interfacial stress due to surface dilatational viscosity. Thus, the dynamics of the interface are determined by the bulk flow field along with the functional form of the surface tension versus surfactant concentration, i.e., the surfactant equation of state, as well as the surface viscosities, which also are functions of the surfactant concentration. If the surfactant system is soluble, then the transport kinetic properties can also play an important role.

In slightly contaminated situations, particularly when the inertial time scales are small compared to the time scales associated with transport kinetics, the gas-liquid interface tends to be swept clean by the bulk flow in the upstream region, and the surfactant molecules tend to accumulate downstream. The separation between clean and surfactant-laden interface tends to be a sharp front. These are physically observed upstream of a surface barrier (4) and computed on rising bubbles (5-8). The location of this surfactant front and the concentration level downstream of the front play a major role in determining the dynamics, not only of the flow at the interface but of the entire coupled interfacebulk flow. The question naturally arises: What determines the location of the front and the surfactant concentration distribution downstream of it? The answer lies in the coupling between the bulk flow, the Marangoni elasticity, and surface viscous and diffusion effects. In order to answer the question, one needs to solve a coupled system, consisting of the Navier-Stokes equations for the bulk flow, advection-diffusion of the (active scalar) concentration of surfactant, and the tangential stress balances at the interface. If the interface is not flat, the normal stress balance also needs to be included.

The present approach is to study a canonical flow geometry using numerical computations of the Navier-Stokes equations. The boundary conditions at the gas-liquid interface are functions of the surface tension and surface viscosities, which in turn are (nonlinear) functions of the surfactant concentration. The geometry is that of the deep-channel surface viscometer, which consists of an annular region bounded by stationary inner and outer cylinders, a rotating floor, and a flat free surface. Here, the channel viscometer is driven relatively fast to produce a secondary flow that drives the fluid at the interface toward the inner cylinder, and yet slow enough to ensure that the flow is laminar. We also have used a depth-to-gap ratio of 0.5 to further 
enhance the secondary flow; when used as a surface viscometer in the Stokes flow regime, $2 / \pi$ is a lower bound for the gap ratio (3). Aside from its general availability, this flow system has the advantage of permitting the relative importance of elasticity and surface viscosity to be controlled by adjusting the rate of rotation, and hence the inertia. The surfactant system modeled in the computations is also based on a physical system, namely a hemicyanine monolayer, which has been studied in several recent fluid flow experiments (9-12).

\section{GOVERNING EQUATIONS}

The flow consists of fluid of density $\rho$ and molecular viscosity $\mu$ (and kinematic viscosity $v=\mu / \rho$ ), contained in an annular region of inner radius $r_{i}$ and outer radius $r_{o}$, filled to a depth $d$. The two cylinder side walls are stationary, and the bottom end wall rotates at a constant rate $\Omega$. The top surface of the fluid is exposed to air and has a surfactant film (insoluble monolayer) on the interface. Initially, everything is at rest, and the surfactant monolayer is uniformly distributed. At time $t=0$, the bottom end wall is impulsively started. A schematic of the flow apparatus is shown in Fig. 1.

The governing equations are the axisymmetric Navier-Stokes equations, together with the continuity equation and appropriate boundary and initial conditions. Using a cylindrical polar coordinate system $(r, \theta, z)$, the Stokes stream function, $\psi$, the axial angular momentum, $\alpha$, and the azimuthal component of vorticity, $\eta$, the nondimensional velocity vector is

$$
\mathbf{U}=(u, v, w)=\left(-\frac{1}{r} \frac{\partial \psi}{\partial z}, \frac{\alpha}{r}, \frac{1}{r} \frac{\partial \psi}{\partial r}\right)
$$

and the corresponding vorticity vector is

$$
\boldsymbol{\nabla} \times \mathbf{U}=\left(-\frac{1}{r} \frac{\partial \alpha}{\partial z}, \eta, \frac{1}{r} \frac{\partial \alpha}{\partial r}\right)
$$

The use of $\psi$ and $\alpha$ is convenient in axisymmetric swirling flows;

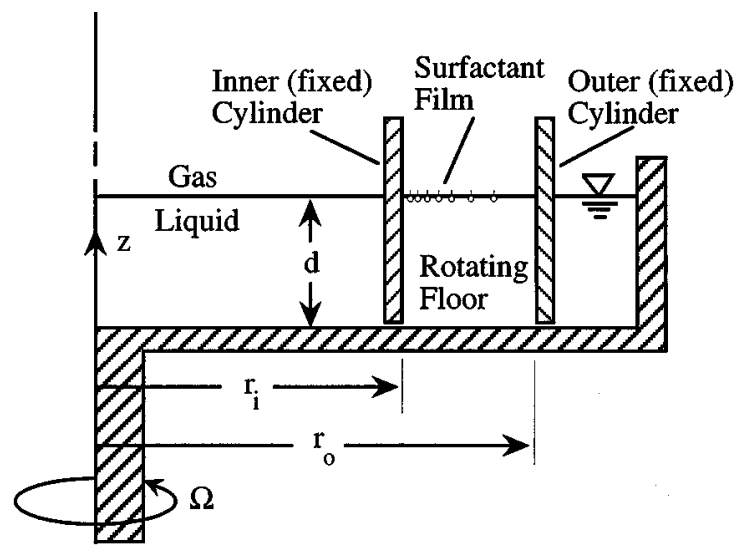

FIG. 1. Schematic of the deep-channel surface viscometer. contours of $\psi$ in an $(r, z)$ plane depict the streamlines of the flow, and contours of $\alpha$ in that plane depict the vortex lines. Recall that vortex lines cannot begin or end at a stationary solid wall, they must be tangential to it, and at a stress-free (e.g., clean) gas-liquid interface the vortex lines are normal to it.

We shall use $r_{o}$ as the length scale and $1 / \Omega$ as the time scale, and define a Reynolds number $R e=\Omega r_{o}^{2} / v$ to nondimensionalize the axisymmetric Navier-Stokes equations:

$$
\begin{gathered}
\frac{D \alpha}{D t}=\frac{1}{R e} \nabla_{*}^{2} \alpha, \\
\frac{D \eta}{D t}+\frac{\eta}{r^{2}} \frac{\partial \psi}{\partial z}-\frac{1}{r^{3}} \frac{\partial \alpha^{2}}{\partial z}=\frac{1}{R e}\left(\nabla^{2} \eta-\frac{\eta}{r^{2}}\right),
\end{gathered}
$$

where

$$
\begin{aligned}
\nabla_{*}^{2} \psi & =-r \eta \\
\frac{D}{D t} & =\frac{\partial}{\partial t}-\frac{1}{r} \frac{\partial \psi}{\partial z} \frac{\partial}{\partial r}+\frac{1}{r} \frac{\partial \psi}{\partial r} \frac{\partial}{\partial z}, \\
\nabla^{2} & =\frac{\partial^{2}}{\partial z^{2}}+\frac{\partial^{2}}{\partial r^{2}}+\frac{1}{r} \frac{\partial}{\partial r}
\end{aligned}
$$

and

$$
\nabla_{*}^{2}=\frac{\partial^{2}}{\partial z^{2}}+\frac{\partial^{2}}{\partial r^{2}}-\frac{1}{r} \frac{\partial}{\partial r} .
$$

The boundary conditions on the solid boundaries are no-slip, i.e., the normal and tangential derivatives of $\psi$ vanish; $\alpha=0$ on the stationary cylinder walls and $\alpha=r^{2}$ on the rotating floor. The azimuthal vorticity $\eta$ on the solid boundaries is determined by evaluating Eq. [3] on the boundaries once $\psi$ is known. On the air-water interface, being a material surface, $\psi$ is continuous with its value on the side walls, which we set to zero without loss of generality. We shall assume that the interface is flat, and hence the contact angle at the air-water-solid contact line is $90^{\circ}$ (in the physical deep-channel viscometer, we fix the location of the contact line by depositing a nonwetting paraffin film above the interface on the cylinder walls; also, the Froude number, $\mathrm{Fr}=$ $\Omega^{2} r_{o}^{2} / g d$, for the range of $R e$ considered is only of order $10^{-2}-$ $\left.10^{-3}\right)$. This leaves the conditions for $\alpha$ and $\eta$ on the interface to be specified.

Our treatment of the interface follows that of Scriven (1), except that we allow the surface viscosities to vary with the surfactant concentration; see $(13,14)$ for a detailed treatment. We also allow for a nonlinear equation of state. For hemicyanine, which forms an insoluble monolayer on the air-water interface, we have measured using standard techniques (Wilhelmy plate measurement with an electrobalance on a Langmuir trough, at $23 \pm 1{ }^{\circ} \mathrm{C}$ ) the surface tension as a function of surfactant concentration up to a surface coverage where the system appears to undergo a phase transition $(10,15)$.

The measured equation of state is presented in Fig. 2a, along with a model that fits the data over a large range of $c$, given by 
(a)

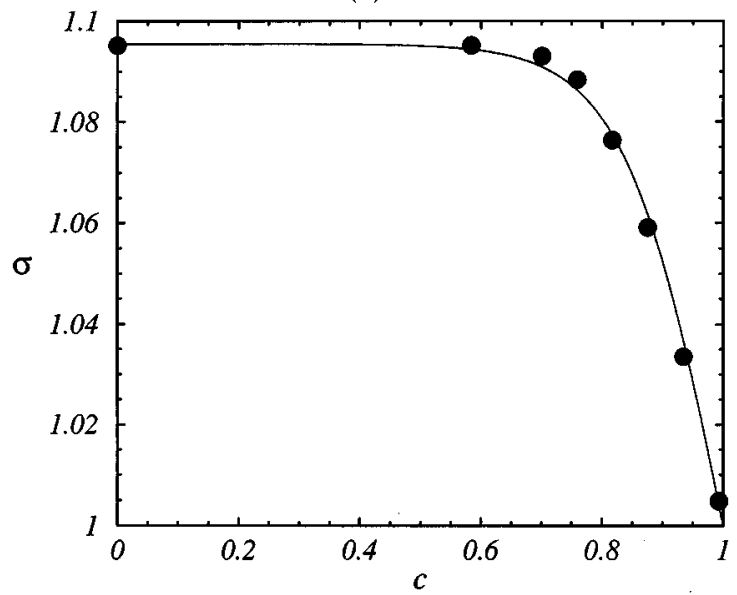

(b)

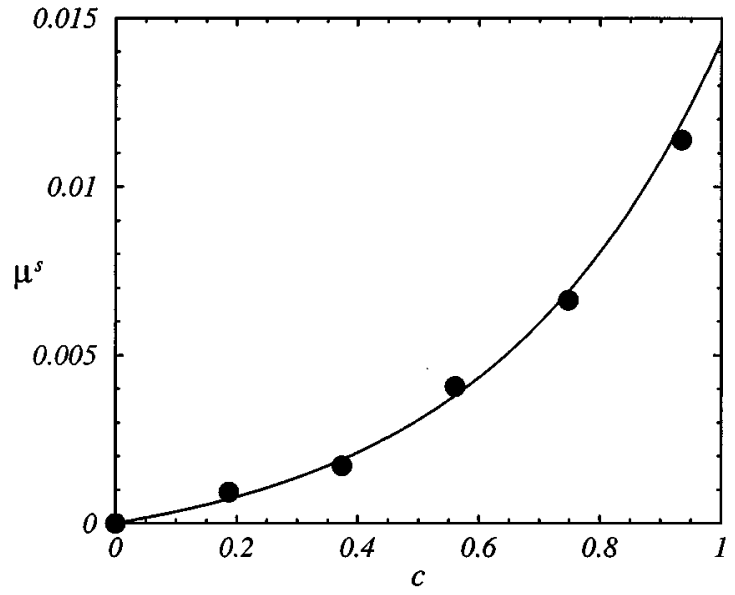

FIG. 2. (a) Equation of state, $\sigma$ vs $c$ (@, experimental measurements of (10); solid line, model Eq. [4]), and (b) surface shear viscosity, $\mu^{s}$ vs $c(\boldsymbol{\bullet}$, experimental measurements; solid line, model Eq. [5]), for hemicyanine at the air-water interface at $23 \pm 1^{\circ} \mathrm{C}$.

the equation

$$
\sigma=1+\frac{a_{1}}{\sigma_{c}} \tanh \left(a_{2}(1-c)\right),
$$

where the surface tension, $\sigma$, has been nondimensionalized with a characteristic surface tension, $\sigma_{c}=66 \mathrm{dyn} / \mathrm{cm}(\mathrm{mN} / \mathrm{m})$, and the surfactant concentration, $c$, has been nondimensionalized with the corresponding characteristic concentration, $c_{c}=$ $0.856 \mathrm{mg} / \mathrm{m}^{2}$. The two parameters, $a_{1}=6.3$ and $a_{2}=6.2$, used to match the model to the data are related to the saturation surface pressure, i.e., the range in surface tension between that of a clean interface and a saturated one (16), and the Marangoni number, which is related to the slope of the equation of state at the inflection point, $\left(\sigma_{c}, c_{c}\right)$, where the phase transition is apparent. The flat behavior of $\sigma$ at small $c$ is representative of insoluble monolayers (16).

The surface viscosities are also nonlinear functions of the thermodynamic state of the interface, namely the surfactant surface concentration and in general the temperature as well, but here we only consider the isothermal case. Using a standard (low $R e$ ) deep-channel surface viscometer (17), we have measured the surface shear viscosity for our surfactant system over the same range of $c$ as given in the equation of state and fitted a model equation to the data (see Fig. 2b). The model equation for the nondimensional surface shear viscosity is

$$
\mu^{s}=1.15 \times 10^{-3}[-1+\exp (2.6 c)],
$$

where $\mu r_{o}$ has been used to nondimensionalize the surface viscosities. The surface dilatational viscosity, $\kappa^{s}$ (nondimensional), is modeled since it has not yet been measured consistently with any two different experimental techniques for any surfactant (3). Measurements of $\kappa^{s}$ for a variety of different surfactants reported in the literature suggest that the value of $\kappa^{s}$ is in general larger than $\mu^{s}$. For example, Avramidis and Jiang (18) report a range of ratios of 2:1-20:1 for a given surfactant, Li and Slattery (19) report a ratio of $2000: 1$, and Maru and Wasan (20) report a range of ratios of $15: 1-400: 1$. The measurements of Hirsa et al. (10) for the sum of the viscosities along with the present measurements of $\mu^{s}$ give a ratio of $1000: 1$. Here, we shall model $\kappa^{s}$ as being 10 times the shear viscosity, $\kappa^{s}=10 \mu^{s}$.

Since the surface tension, $\sigma$, and the surface viscosities, $\mu^{s}$ and $\kappa^{s}$, are all functions of the surfactant concentration $c$, we need to solve an active scalar advection-diffusion equation for $c$ :

$$
\frac{\partial c}{\partial t}=\frac{1}{r} \frac{\partial}{\partial r}\left(c \frac{\partial \psi}{\partial z}\right)+\frac{1}{P e^{s}}\left(\frac{\partial^{2} c}{\partial r^{2}}+\frac{1}{r} \frac{\partial c}{\partial r}\right),
$$

where $P e^{s}=\Omega r_{o}^{2} / D^{s}$ is the surface Peclet number and $D^{s}$ is the surface diffusion of the surfactant; $D^{s}$ is estimated to be of order $10^{-5} \mathrm{~cm}^{2} / \mathrm{s}$ for typical surfactants (21). For hemicyanine, Hirsa et al. (10) estimated an upper bound $D^{s}<10^{-6} \mathrm{~cm}^{2} / \mathrm{s}$. For $R e=2000, \Omega r_{o}^{2}=20 \mathrm{~cm}^{2} / \mathrm{s}$, and in the computations we use $P e^{s}$ up to $5 \times 10^{4}$; this upper value is essentially determined by the available grid resolution. At the contamination front, the large gradients in concentration lead to a large production in surface azimuthal vorticity, resulting in a spike in $\eta$ whose width scales with $1 /\left(P e^{s}\right)^{1 / 2}$. For a fixed finite difference grid, there is a maximum $P e^{s}$ that can be resolved.

For a flat interface, only the tangential stress balance plays a dynamic role. The tangential stress balance in the azimuthal direction is

$$
\frac{\partial \alpha}{\partial z}=\mu^{s}\left(\frac{\partial^{2} \alpha}{\partial r^{2}}-\frac{1}{r} \frac{\partial \alpha}{\partial r}\right)+\frac{\partial \mu^{s}}{\partial r}\left(\frac{\partial \alpha}{\partial r}-\frac{2 \alpha}{r}\right),
$$

and in the radial direction

$$
\begin{aligned}
\eta= & \frac{1}{C a} \frac{\partial \sigma}{\partial r}+\left(\mu^{s}+\kappa^{s}\right)\left(\frac{1}{r^{2}} \frac{\partial^{2} \psi}{\partial r \partial z}-\frac{1}{r} \frac{\partial^{3} \psi}{\partial r^{2} \partial z}\right) \\
& -\frac{1}{r} \frac{\partial^{2} \psi}{\partial r \partial z} \frac{\partial\left(\mu^{s}+\kappa^{s}\right)}{\partial r}+\frac{2}{r^{2}} \frac{\partial \psi}{\partial z} \frac{\partial \mu^{s}}{\partial r},
\end{aligned}
$$


where $C a=\mu \Omega r_{o} / \sigma_{c}$ is the capillary number. Both Eqs. [7] and [8], derived in (14), are solved at the interface.

\section{NUMERICAL TECHNIQUE}

Due to the nonlinear coupling between the bulk flow and the boundary conditions, an explicit time integration is implemented. We begin by discretizing in space using second-order centered differences. Equations [1] and [2] then have the form

$$
\frac{d \alpha_{i, j}}{d t}=R H S_{1}(\alpha, \psi)
$$

and

$$
\frac{d \eta_{i, j}}{d t}=R H S_{2}(\eta, \alpha, \psi)
$$

The computational domain is $r \in\left[r_{i} / r_{o}, 1\right], z \in\left[0, d /\left(r_{o}-r_{i}\right)\right]$ with $r=r_{i} / r_{o}+i\left(r_{o}-r_{i}\right) / r_{o} n_{r}$ for $i \in\left[0, n_{r}\right]$ and $z=j d /$ $r_{o} n_{z}$ for $j \in\left[0, n_{z}\right]$ (for the results presented here, $n_{r}=200$, $n_{z}=100, \delta t=10^{-4}, d /\left(r_{o}-r_{i}\right)=0.5$, and $\left.r_{i} / r_{o}=0.7865\right)$.

Starting from the initial conditions, the interior values of $\alpha_{i, j}$ and $\eta_{i, j}\left(i \in\left[1, n_{r}-1\right]\right.$ and $\left.j \in\left[1, n_{z}-1\right]\right)$ are evolved forward in time using a second-order predictor-corrector scheme. Denoting the current time by superscript $k$, the predictor stage by $\operatorname{superscript} *$, and the next (corrected) stage by $k+1$, we first evaluate

$$
\alpha_{i, j}^{*}=\alpha_{i, j}^{k}+\delta t R H S_{1}^{k}
$$

and

$$
\eta_{i, j}^{*}=\eta_{i, j}^{k}+\delta t R H S_{2}^{k}
$$

At this stage, we need to solve the elliptic equation [3] for $\psi^{*}$ with the interior points for $\eta^{*}$ just computed. Then, the surfactant concentration is advected by this streamfunction. So Eq. [6] is solved for $c^{*}$ with $\psi^{*}$, and the boundary conditions $\partial c / \partial r=0$ at $r=r_{i} / r_{o}$ and 1 (thus conserving total surfactant on the interface). This evolution is also done by the predictor-corrector scheme. One needs to do the full two stages to get from $c^{k}$ to $c^{* *}$ to $c^{*}$, both stages using $\psi^{*}$. With $c^{*}(r)$, we evaluate $\sigma\left(c^{*}(r)\right)$, $\mu^{s}\left(c^{*}(r)\right), \kappa^{s}\left(c^{*}(r)\right)$, and their radial derivatives. The boundary conditions for $\alpha^{*}$ and $\eta^{*}$ are then evaluated. On the no-slip boundaries, this is straightforward. For the interface, we require the normal derivatives of $\psi$ and $\alpha$ at the interface (obtained using one-sided differences) and its radial variations, and then solve Eqs. [7] and [8]. Equation [7] is a second-order ODE for $\alpha$ at the interface, which reduces to a tridiagonal solve. Equation [8] is a straightforward evaluation now that we have the surface tension gradient and surface viscosities and their gradients. We now have everything ( $\alpha, \eta, \psi$, and $c$ ) at the predictor stage and can repeat the whole process on everything to obtain the corrector stage. We evolve $\alpha$ and $\eta$ using

$$
\alpha_{i, j}^{k+1}=0.5\left(\alpha_{i, j}^{k}+\alpha_{i, j}^{*}+\delta t R H S_{1}^{*}\right)
$$

and

$$
\eta_{i, j}^{k+1}=0.5\left(\eta_{i, j}^{k}+\eta_{i, j}^{*}+\delta t R H S_{2}^{*}\right)
$$

\section{RESULTS}

The results presented here are in an annular channel with the geometry fixed by $r_{i} / r_{o}=0.7865$ and $d /\left(r_{o}-r_{i}\right)=0.5$. The surfactant system corresponds to a hemicyanine monolayer on a water substrate at $23 \pm 1{ }^{\circ} \mathrm{C}$, and this fixes the material properties. We consider a range of initial uniform surfactant surface concentration, $0 \leq c_{0} \leq 0.7$ (this range together with our other choices of parameters ensures that $c(r, t)<1.0)$. The two dynamical variables $R e$ and $C a$ depend linearly on the rate of rotation of the floor, $\Omega$. We have considered two rotation rates corresponding to flow states beyond the Stokes flow regime where the flow is nonlinear and yet steady and axisymmetric, i.e., $R e=2000, C a=1 / 3630$ and $R e=8000, C a=4 / 3630$.

A contamination front only forms when there is a balance between the interfacial viscoelasticity and the bulk flow near the interface. In the absence of the viscoelasticity, the surfactant molecules would act as passive scalars, advected by the bulk flow at the interface, and so would accumulate at the inner cylinder wall since they are insoluble. For a description of the flow in this geometry, in the absence of surfactants, see (13).

There are three mechanisms at play that prevent such a buildup at the inner cylinder wall, each of which dominates at different concentration and concentration gradient levels. If the concentration levels tend to become large (as they would be if the surfactants started to pile up at the inner cylinder), then their finite diffusivity, $P e^{s}$, would oppose such a buildup, smearing them out over a length that scales with $1 /\left(P e^{s}\right)^{1 / 2}$. Such a mechanism would also be present if $c$ were a passive scalar. However, $c$ is an active scalar that determines the surface tension and surface viscosities (through Eqs. [4] and [5]), which in turn determine the stress balances at the interface that feed into the bulk flow as boundary conditions for the vorticity and angular momentum. This then introduces two additional mechanisms which resist the surfactant pileup and also tend to smear out any contamination front. The surface viscosities will tend to oppose the buildup of surfactant by the bulk flow compression of the interface; this is triggered not only by the concentration gradient but also by the concentration level (see Eq. [8]). Figure $2 b$ shows how $\mu^{s}$ increases exponentially with $c$ for hemicyanine. The third mechanism, which in the cases presented here is dominant, is the Marangoni stress $\partial \sigma / \partial r$, which imparts an elasticity to the interface that resists the buildup of surfactants due to the stretching and compacting of the interface by the bulk flow. Eggleton et al. (22) present a detailed exposition on how this stress functions in an insoluble system and how the packing of 
(a)

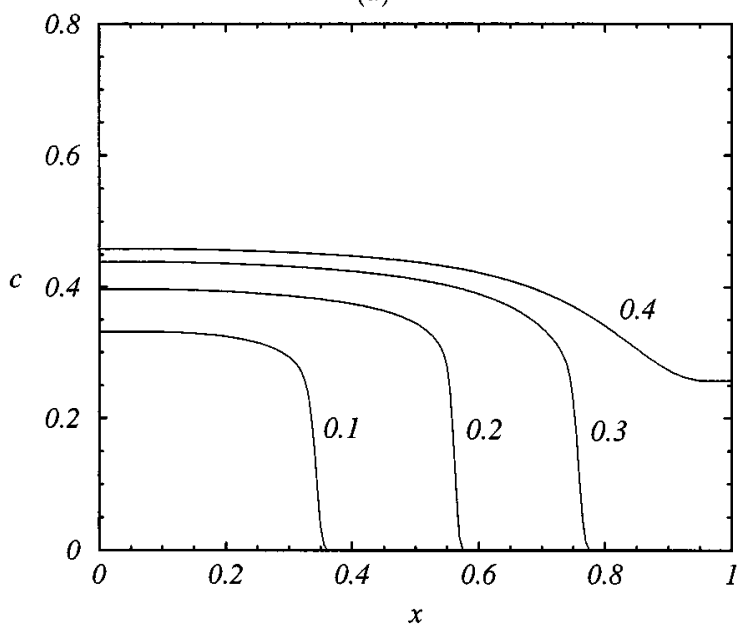

(b)

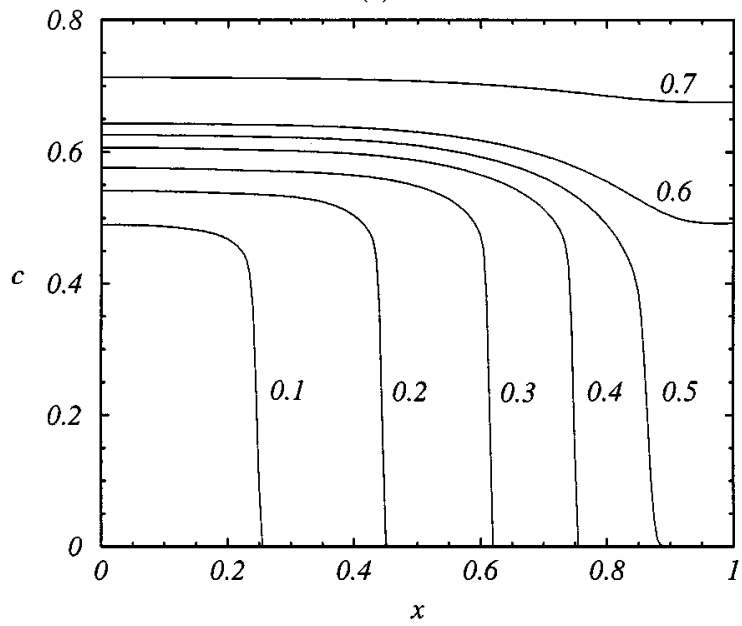

FIG . 3. Surfactant concentration profiles, $c$, at steady state, with initial uniform distribution $c=c_{0}$, for $c_{0}$ as indicated: (a) $R e=2000, C a=1 / 3630$, (b) $R e=8000, C a=4 / 3630$.

surfactant molecules leads to a nonlinear equation of state. For the insoluble system being studied here, once the concentration level drops below a certain level (about $c=0.3$ ), the Marangoni stress mechanism essentially vanishes (see Fig. 2a), allowing the surface to be cleaned by bulk flow advection. As the concentration builds up at small radii, the Marangoni stress rapidly increases, and this mechanism tends to smear the surfactants out toward a uniform distribution. So we find that the contamination front essentially forms where the competition between the bulk flow advection and the Marangoni stress just balance, and this for our bounded, insoluble system depends critically on the total amount of surfactant residing on the interface. We measure this by $c_{0}$, the initial uniform concentration distribution.

Figures 3 and 4 show the steady-state concentration distributions and azimuthal vorticity profiles at the interface as functions of the dimensionless gap coordinate, $x=\left(r-r_{i}\right) /\left(r_{o}-r_{i}\right)$, that result for different values of $c_{0}$. We find that if the initial con- centration level is very low, the Marangoni stress is essentially zero, the bulk flow advects all the surfactant in toward the inner cylinder, and the surface diffusion (and to a lesser extent the surface viscosity) mechanism determines the equilibrium surfactant distribution. For $R e=2000$, Figs. $3 \mathrm{a}$ and $4 \mathrm{a}$ show that a contamination front forms for $c_{0}<0.4$, with a corresponding surface boundary layer. For $c_{0}<0.4$, the Marangoni stress dominates in determining the steady-state $c$ distribution, and the radial location of the contamination front varies almost linearly with $c_{0}$. For $c_{0}$ above about 0.4 , the Marangoni stress is so large that it completely dominates, not only the surface diffusion and viscosities but also the bulk flow, and it almost completely resists the formation of surfactant gradients. For larger $R e$, the secondary flow is stronger and the $c_{0}$ range over which a contamination front can form is extended. Figures $3 \mathrm{~b}$ and $4 \mathrm{~b}$ show that for $R e=8000$ the secondary flow can partially clean the interface for $c_{0}<0.6$. With the stronger secondary flow at $R e=8000$, the variation of the front location is no longer linear with $c_{0}$. A further

(a)

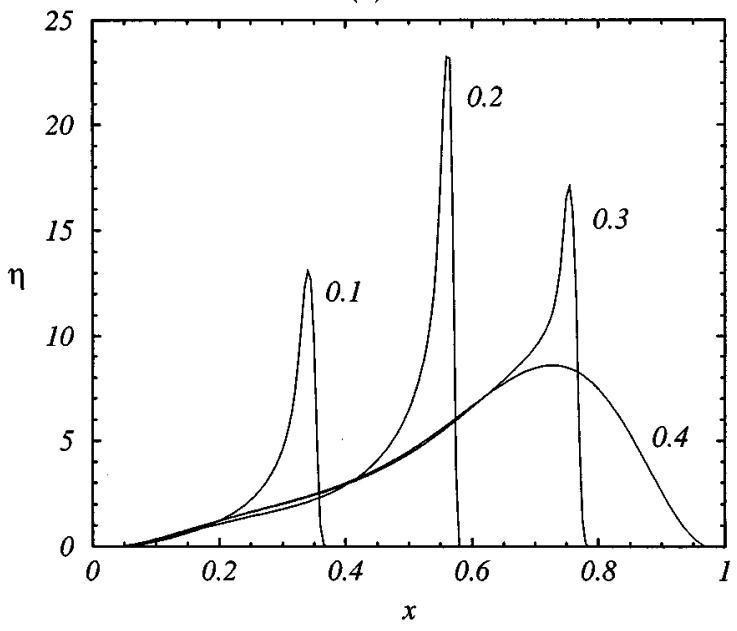

(b)

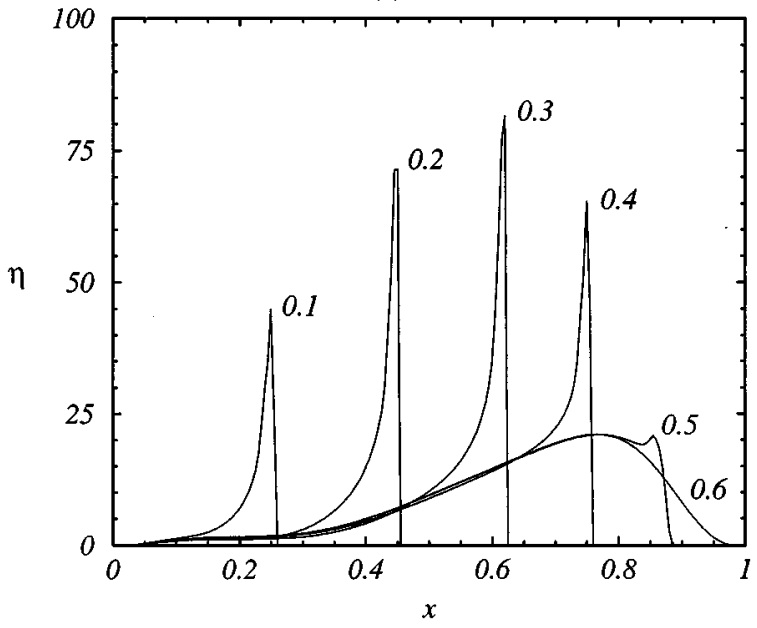

FIG. 4. Surface azimuthal vorticity profiles, $\eta$, at steady state for $c_{0}$ as indicated: (a) $R e=2000, C a=1 / 3630$; (b) $R e=8000, C a=4 / 3630$. 
contribution to the overall nonlinearity of the flow is due to the fact that larger $c_{0}$ leads to values of $c$ where the equation of state manifests greater nonlinearity. Qualitatively similar results have been obtained by Eggleton et al. (22) in the inertialess limit, but with highly deformable interfaces.

Typically, when the Marangoni stress is dominating, the interface is thought of as immobile, acting as a no-slip surface. But this is only true for the velocity components in the directions of the Marangoni stress. In this axisymmetric swirling flow, this direction is radial. In the azimuthal direction, since the flow is axisymmetric, there are no azimuthal gradients of surfactant (or anything else for that matter), and so there are no Marangoni stresses acting in that direction. The result is that in the radial direction, the Marangoni stress makes the interface act like a no-slip surface, but in the azimuthal direction it is not immobilized. In both directions, there are some contributions from the surface viscosities, and we explore these in more detail below. Figures 5 and 6 illustrate that behind the contamination

(a)

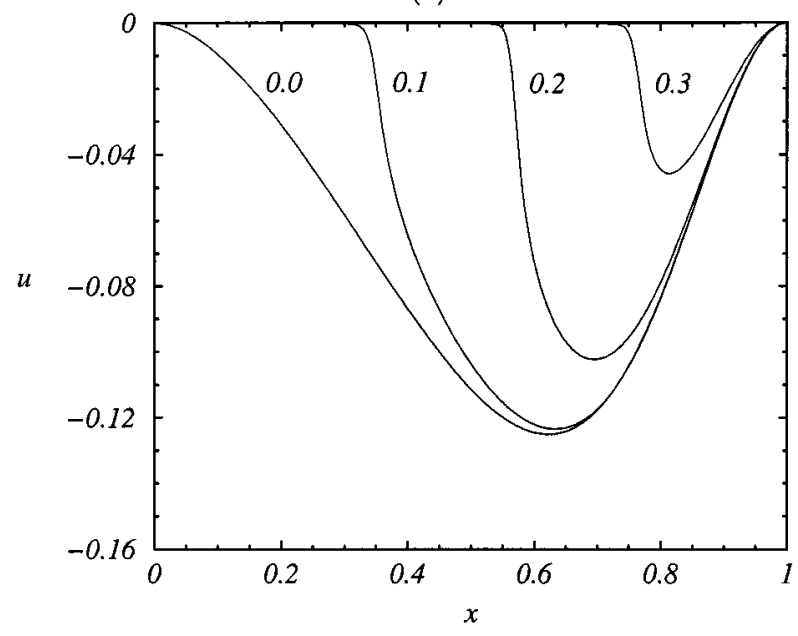

(b)

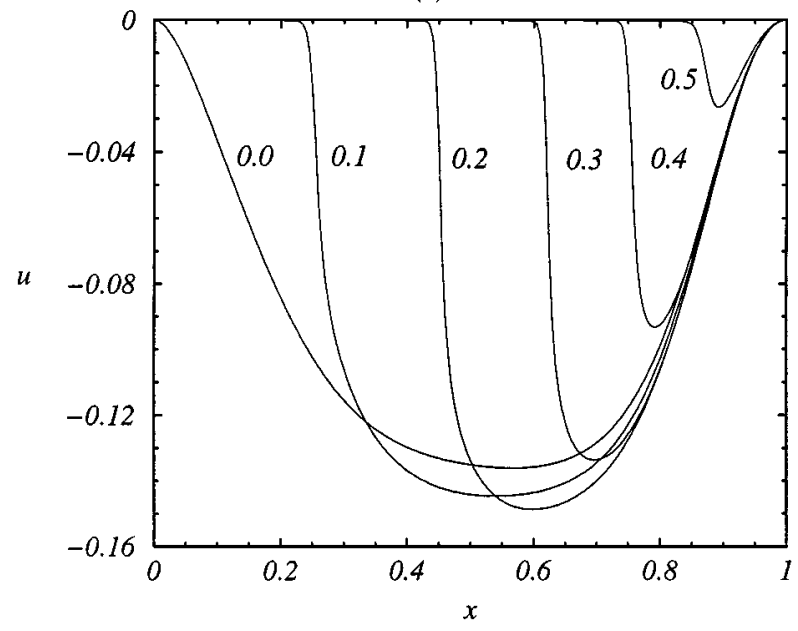

FIG . 5. Surface radial velocity profiles, $u$, at steady state for $c_{0}$ as indicated: (a) $R e=2000, C a=1 / 3630$; (b) $R e=8000, C a=4 / 3630$. (a)

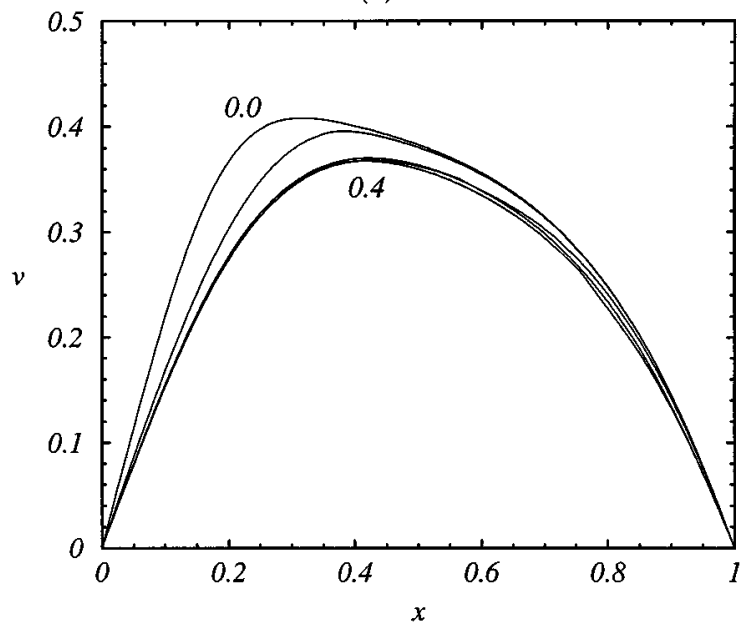

(b)

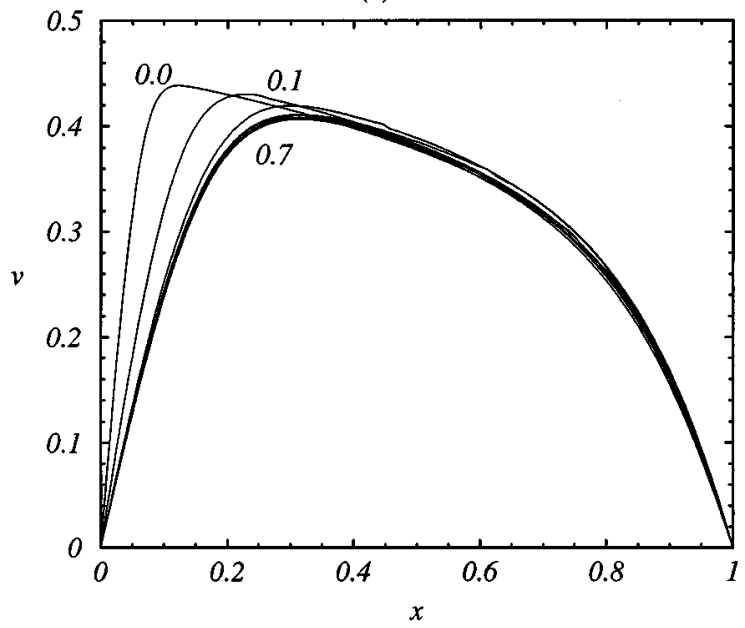

FIG. 6. Surface azimuthal velocity profiles, $v$, at steady state for $c_{0}$ as indicated: (a) $R e=2000, C a=1 / 3630$; (b) $R e=8000, C a=4 / 3630$.

front, the radial surface velocity, $u$, vanishes, yet the surface azimuthal velocity is only slightly changed from that of the clean surface $\left(c_{0}=0\right)$. This has fundamental consequences for models of contaminated interfaces that are not two-dimensional; surfactant coverage does not simply mean that the interface is no-slip.

It should be noted that over the surfactant-covered region, where the radial velocity $u=-1 / r \partial \psi / \partial z=0$, Eq. [8] indicates that the only nonzero contribution to the radial stress balance, and thus $\eta$ at the interface, is from the Marangoni stress. Thus, the surface viscosity terms on the right-hand side of Eq. [8] can only contribute to the surface vorticity during the transient evolution to steady state and vanish at steady state. Note, however, that immediately behind the contamination front the radial velocity, $u$, smoothly drops from its maximum value to zero over a region that scales with the width of the spike in the surface azimuthal vorticity, $\eta$. In this region, where the effects of finite surface diffusivity are present, the surface viscosities can 
make a contribution to the radial stress balance since the surface velocity gradients are nonzero.

The above observations motivate us to consider three different idealizations of the interfacial stress balance to explore what role the nonlinear equation of state and the surface viscosities play in determining the coupled interfacial-bulk flow. The stress balance described by the full formulation (Eqs. [7] and [8]) will be referred to as the Viscoelastic interface. The idealizations are (i) Elastic $\left(\mu^{s}=\kappa^{s}=0\right)$, where $\eta=1 / C a \partial \sigma / \partial r$ and $\partial \alpha / \partial z=$ 0; (ii) Pseudo-Visc, where $\eta=1 / C a \partial \sigma / \partial z$, but $\alpha$ satisfies [7]; and (iii) Pseudo-Stag, where $\eta$ satisfies the condition for a rigid, no-slip surface, $\eta=-1 / r \partial^{2} \psi / \partial z^{2}$, but $\alpha$ satisfies [7]. We include for comparison purposes the case of a no-slip solid wall (Rigid), where $\eta=-1 / r \partial^{2} \psi / \partial z^{2}$ and $\alpha=0$. Figure 7 presents the profiles of $\eta$ and $v$ at the interface using these conditions. Figure 8a shows the relative errors in $\eta$ across the interface be-

(a)

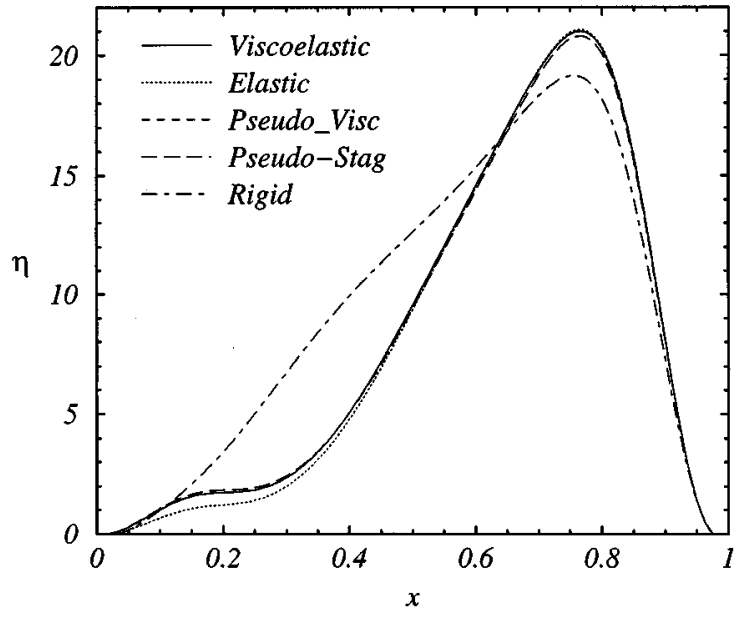

(b)

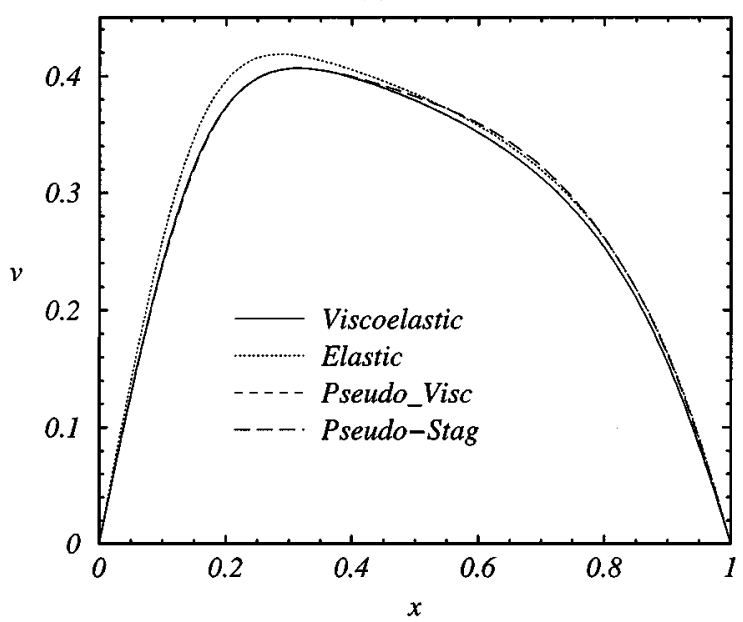

FIG. 7. Profiles of the interfacial values of (a) $\eta$ and (b) $v$ for the various interfacial conditions as indicated for $c_{0}=0.7, R e=8000$, and $C a=4 / 3630$. Note that (i) the $\eta$ profiles for Viscoelastic and Pseudo-Visc virtually coincide and (ii) the $v$ profile for Rigid is not drawn since it is identically zero. (a)

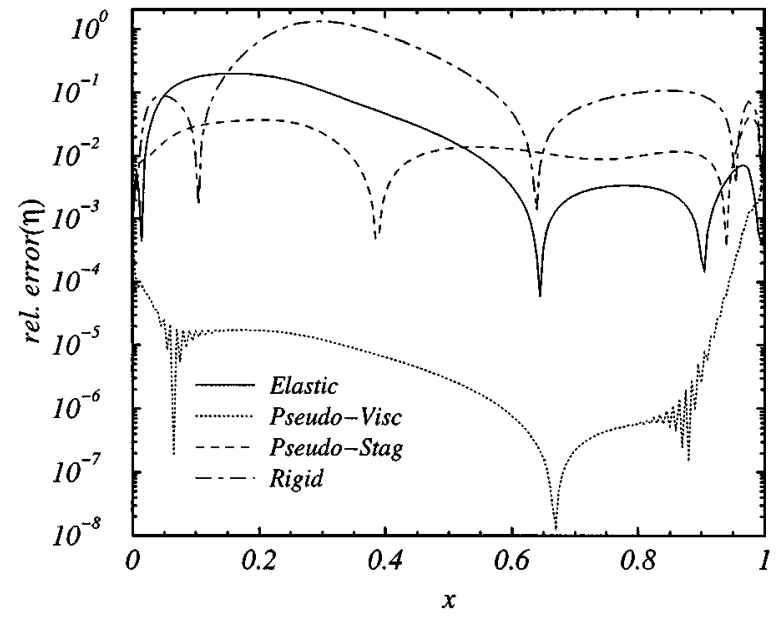

(b)

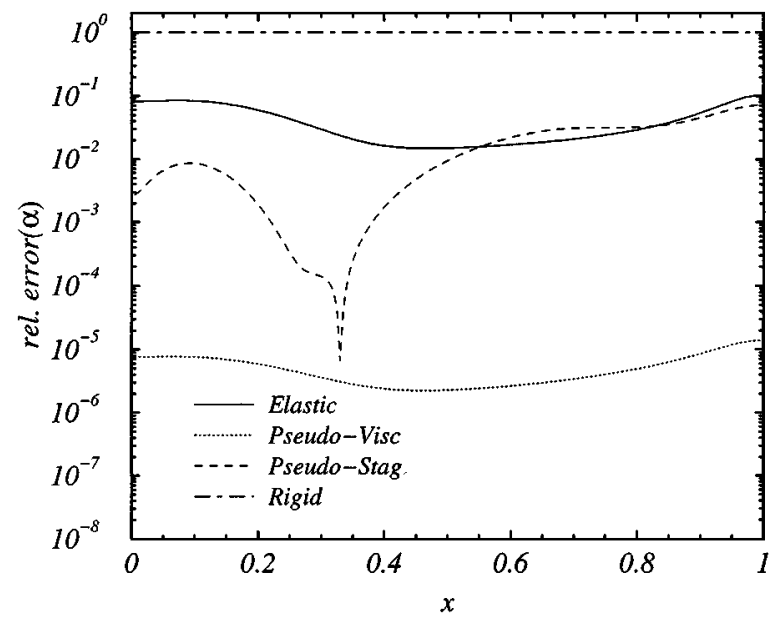

FIG. 8. Relative errors in the interfacial values of (a) $\eta$ and (b) $\alpha=r v$, between the various interfacial conditions shown in Fig. 7 and the Viscoelastic condition, as indicated, for $c_{0}=0.7, R e=8000$, and $C a=4 / 3630$.

tween using the full viscoelastic conditions and each of the idealizations and the rigid wall condition for the $R e=8000$ flow with $c_{0}=0.7$. In Fig. $8 \mathrm{~b}$, the corresponding relative errors in $\alpha=r v$ are shown.

A comparison of the various relative errors leads to the following observations:

1. The results using the Viscoelastic and the Pseudo-Visc conditions are virtually indistinguishable. This is because the surface viscosities play no role in the radial stress balance at steady state since the radial velocity vanishes on the monolayer which covers the entire interface.

2. The comparison between the Elastic and the Viscoelastic cases shows marked differences; we have already observed from the comparison with the Pseudo-Visc condition that surface viscosities do not play a major role in the radial stress balance for this flow situation, but having dropped the surface shear 
viscosity to zero in the azimuthal stress balance causes discrepancies of order $10 \%$.

3. Replacing the radial stress balance (in effect the details of the nonlinear equation of state) with the radial stress corresponding to a no-slip wall in the Pseudo-Stag case results in smaller discrepancies than neglecting $\mu^{s}$ in the azimuthal stress balance (Elastic). Of course, this is so only when $c_{0}$ is sufficiently large that the bulk flow does not manage to clean any part of the interface. If $c_{0}$ is sufficiently small (in this case $<0.6$ ), the Pseudo-Stag condition is incapable of determining how the interface is to be cleaned since it is missing the correct Marangoni stress contribution.

4. The Rigid case fails to capture the correct interfacial conditions. The relative error in $\alpha$ is $100 \%$. Even though the condition on $\eta$ is the same as that used in the Pseudo-Stag case, which gave a reasonably close comparison with the true viscoelastic conditions, the large errors in $\alpha$ also contribute to errors in $\eta$ via the $-1 / r^{3} \partial \alpha^{2} / \partial z$ term in Eq. [2].

Observation 4 deserves further comment. It has been widely observed that in many interfacial flows, replacing the tangential stress balance with the stress due to a rigid wall is an approximation that works very well when the interface has high surface coverage (this is the basis for the well-known stagnant cap approximation). However, in all the cases where this limit has worked, the flow has either been planar two-dimensional or axisymmetric with no swirl. In these cases, the vorticity vector has only one nonzero component, that in the direction perpendicular to the velocity vector (which only has two nonzero components), and the only nontrivial tangential stress balance is in the direction perpendicular to the vorticity. In that direction, the stress balance consists of two contributions from the presence of surfactants, the surface tension gradient (scaled by $1 / \mathrm{Ca}$ ) and surface viscous effects (scaled by the Boussinesq number, $\mu^{s}+\kappa^{s}$ ). In most of the flow situations studied, the surface tension gradient dominates the stress balance in this direction (as is the case in our problem), and the rigid approximation works very well, at least in the steady-state regimes studied. What distinguishes our problem is that it involves flow that is axisymmetric with swirl, the velocity component in the $\theta$ direction is nonzero, and hence the vorticity vector has three nonzero components. For a complete tangential stress balance at the interface, the balance in the $\theta$ direction (Eq. [7]) must be included. In this direction, there is no direct contribution from the surface tension gradient; this component of the stress balance is dominated by surface shear viscosity effects. Recent experiments (9) using a flow which initially only has nonzero velocity in the $\theta$ direction demonstrated the profound influence $\mu^{s}$ can have on the air-water interface. When there are flow directions in which there are no concentration gradients, then regardless of how heavily loaded with surfactants the interface is, the stress balance in these directions is dominated by surface shear viscosity effects and the rigid approximation fails to capture this. The rigid approximation only works well in capturing the surface tension gradient contribution at steady state.

\section{CONCLUSION}

A fully coupled viscoelastic interfacial flow with a swirling bulk flow has been modeled numerically for flow and material properties corresponding to a physical air-water system with a hemicyanine monolayer. For the present numerical calculations, the equation of state, $\sigma(c)$, and surface shear viscosity, $\mu^{s}(c)$, were measured in the laboratory for the surfactant modeled in this study. The calculations show that the surfactant distribution and the stress balance are critically dependent on the form of $\sigma(c)$, which can vary significantly between different classes of surfactants, namely, gas-, liquid-, or solid-like surfactant systems $(16,23)$, as well as between different surfactants in a given class. In the radial direction, the tangential stress balance for this system was dominated by the Marangoni stress, due in part to the radial velocity diminishing to zero at steady state, where the interface had surfactant coverage, and also due to the product $\left(\mu^{s}+\kappa^{s}\right) C a$ being small $\left(\sim 10^{-6}\right)$. However, in the azimuthal direction, the tangential stress balance is solely determined by the finite surface shear viscosity, $\mu^{s}$. This is due to the flow being axisymmetric and hence having no gradients in the azimuthal direction. Since the surface dilatational viscosity, $\kappa^{s}$, only appears in the radial stress balance, the present flow is insensitive to $\kappa^{s}$ at steady state. Work is underway to explore unsteady regimes where the elastic and viscous terms are more comparable, and in these unsteady viscoelastic regimes we hope to be able to estimate the surface dilatational viscosity.

\section{ACKNOWLEDGMENTS}

We thank C. M. Tomaso for making the measurements of $\mu^{s}$, the surface shear viscosity. This work was supported by NSF Grants CTS-9803478 and CTS-9896259.

\section{REFERENCES}

1. Scriven, L. E., Chem. Eng. Sci. 12, 98 (1960).

2. Aris, R., "Vectors, Tensors, and the Basic Equations of Fluid Mechanics." Prentice-Hall, Englewood Cliffs, NJ, 1962.

3. Edwards, D. A., Brenner, H., and Wasan, D. T., "Interfacial Transport Processes and Rheology.” Butterworth-Heinemann, Boston, 1991.

4. Scott, J. C., J. Fluid Mech. 116, 283 (1982).

5. Davis, R. E., and Acrivos, A., Chem. Eng. Sci. 21, 681 (1966).

6. Sadhal, S. S., and Johnson, R. E., J. Fluid Mech. 126, 237 (1983).

7. He, Z., Maldarelli, C., and Dagan, Z., J. Colloid Interface Sci. 146, 442 (1991).

8. Bel Fdhila, R., and Duineveld, P. C., Phys. Fluids 8, 310 (1996).

9. Hirsa, A., Harper, J. E., and Kim, S., Phys. Fluids 7, 2532 (1995).

10. Hirsa, A., Korenowski, G. M., Logory, L. M., and Judd, C. D., Langmuir 13, 3813 (1997).

11. Hirsa, A., Korenowski, G. M., Logory, L. M., and Judd, C. D., Exp. Fluids 22, 239 (1997).

12. Hirsa, A., Vogel, M. J., and Gayton, J. D., Exp. Fluids (2000), in press.

13. Lopez, J. M., and Hirsa, A., J. Colloid Interface Sci. 206, 231 (1998).

14. Lopez, J. M., and Chen, J., J. Fluids Eng. 120, 655 (1998). 
15. Judd, C. D., "Nonlinear Optical Spectroscopy of the Air/Water Interface," Ph.D. Thesis, Rensselaer Polytechnic Institute, 1996.

16. Gains, G. L., "Insoluble Monolayers at Liquid-Gas Interfaces." Interscience, New York, 1966.

17. Mannheimer, R. J., and Schechter, R. S., J. Colloid Interface Sci. 32, 195 (1970).

18. Avramidis, K. S., and Jiang, T. S., J. Colloid Interface Sci. 147, 262 (1991).
19. Li, D., and Slattery, J. C., AIChE J. 34, 862 (1988).

20. Maru, H. C., and Wasan, D. T., Chem. Eng. Sci. 34, 1295 (1979).

21. Agrawal, M. L., and Neuman, R. D., J. Colloid Interface Sci. 121, 366 (1988).

22. Eggleton, C. E., Pawar, Y. P., and Stebe, K. J., J. Fluid Mech. 385, 79 (1999).

23. Adamson, A. W., "Physical Chemistry of Surfaces." Wiley-Interscience, New York, 1990. 